\title{
METHOD OF PARTICLE CHARACTERISATION; MORPHOLOGY BY IMAGE ANALYSIS
}

\author{
K. Belaroui ${ }^{1 *}$ and M. N Pons ${ }^{2}$ \\ ${ }^{1}$ Laboratoire STGP, Université des Sciences et de la Technologie Med-BOUDIAF d'Oran \\ (USTO-MB) B.P.1505 - EL M'nouer Oran (Algérie). \\ ${ }^{2}$ Laboratoire Réactions et Génie des Procédés (UPR 3349 CNRS) 1 rue Grandville, BP 20451, \\ F-54001 Nancy Cedex, France
}

Received: 19 October 2012 / Accepted: 21 December 2012 / Published online: 31 December 2012

\begin{abstract}
The aim of our work is to establish a morphological characterization of particles. The particle size analysis determines the size distribution of a population of particles using a laser granulometer device. The evolution of particle size distributions is complemented by a study on the particle shape. Thus, morphological analysis consists to acquire images of the particles by using a scanning electron microscopy which will be then processed using an algorithm. The treatment is a set of operations that are thresholding, erosion, expansion and reconstruction of the image. Data processing is performed based on a set of dimensional parameters.
\end{abstract}

Keywords: Characterization; particle size; morphology; image analysis; porous media

\section{INTRODUCTION}

La puissance de l'analyse d'images comme méthode d'évaluation de la forme et de la taille des particules peut être d'un grand secours pour améliorer les procédés de production, en plus d'offrir la possibilité de maximiser la performance des produits.

Author Correspondence, e-mail: kbelaroui@yahoo.fr

ICID: 1025912 
L'utilisation de l'analyse d'images combinée avec la microscopie optique ou électronique est de plus en plus répandue en tant qu'une des méthodes les plus fiables pour mesurer et caractériser les distributions de tailles, de formes et de volumes des particules. L'analyse d'images a aussi l'avantage de générer de l'information détaillée sur les particules individuelles.

Le but de l'étude consiste à étudier la granulométrie et la morphologie de différentes poudres. L'étude de la morphologie est faite par le biais de l'analyse d'images. Elle consiste à acquérir des images des particules qui sont ensuite traitées à l'aide d'un algorithme qui effectue une suite d'instruction permettant l'obtention des images sur lesquelles les mesures vont être effectuées. Ces mesures permettent l'obtention des différents facteurs macroscopiques caractérisant la morphologie et donc la forme des particules.

Un certain nombre de grandeurs physiques et chimiques permettent de caractériser les grains constitutifs d'une poudre. Dans cette étude nous nous intéressons plus particulièrement aux paramètres morphologiques. Les plus importantes sont la taille et la forme. La description de la taille est fortement liée à celle de la morphologie. En effet, la sphère est le seul objet dont la taille puisse être caractérisée par un seul nombre, le diamètre. Suivant la technique de mesure de taille utilisée, on observera une interférence plus ou moins grande de la forme [1].

\section{ANALYSE MORPHOLOGIQUE}

\subsection{Analyse d'images}

L'analyse d'images permet la quantification de l'information contenue dans une image et rend possible une description et une reconnaissance précise, reproductible, éventuellement automatique d'une structure ou d'un ensemble d'éléments. Elle est particulièrement adaptée à l'étude des échantillons microscopiques qu'elle permet de mesurer. Il s'agit de quantifier la forme des objets au moyen de paramètres géométrique dans le but d'établir des relations entre des paramètres morphologiques et des paramètres physiques, chimique, mécaniques,...

Une image est une représentation bidimensionnelle d'un objet et est obtenue à un grossissement donné. Il s'agit au départ dans chaque cas de transformer une image analogique en une image numérique au moyen d'un convertisseur analogique digital (A/D).

Les points de l'image sont disposés suivant une grille en réseau carré, ou les points de la grille représentent des pixels. Plus il y a de pixels plus on a besoin d'une mémoire importante : ainsi une image de 512 lignes de 512 pixels acquise sur 8 bits nécessite 256 Koctets de mémoire. 
Chaque pixel est affecté d'une valeur variant de 0 à 255 , ce que l'on appelle un niveau de gris [2-3].

L'analyse d'images comporte trois étapes :

1. acquisition de l'image

2. traitement de l'image

3. mesures

1. Acquisition :

Le dispositif d'acquisition doit être adapté à chaque situation. Les analyseurs peuvent être reliés à des microscopes optiques (à transmission, à réflexion ou inverse), à des microscopes électroniques (à balayage ou à transmission). Ils permettent aussi, par une camera équipée d'un objectif macro, de reprendre des documents photographiques, des clichés radiologiques ou autoradiographiques, et toutes sortes d'objets macroscopiques (poudres, grains,...) [3-4]. Nous utilisons un microscope électronique à balayage et un microscope optique pour l'obtention de nos images.

\section{Traitement :}

Le traitement peut être fait sur des images en niveaux de gris ou sur des images binaires. Il permet aussi l'obtention des secondes à partir des premières. C'est ce que l'on appelle le seuillage ou la binarisation. Une image binaire est une image dans laquelle les pixels ne peuvent avoir que deux valeurs : 0 et 1 . Par convention, les pixels de valeur 1 seront les pixels objets et appartiendront aux objets, c'est-à-dire aux zones retenues pour l'étude, les pixels de valeur 0 constituant le fond des images binaires. Le seuillage est une opération très délicate qui conditionne le résultat final de l'analyse d'images, il peut être manuel, c'est-à-dire effectué par l'operateur pour chaque image. Dans le premier cas sa réussite est liée à l'objectivité de l'être humain, en particulier à son état de fatigue. L'automatisation est donc préférable. De nombreuses méthodes existent et actuellement la plupart des logiciels d'analyse d'images proposent, en standard, des seuillages basés sur les propriétés de l'histogramme de l'image, c'est-à-dire de la distribution de ses niveaux de gris [4].

Le choix de la méthode dépend de la qualité de l'image. Si une série de clichés a été obtenue pour des conditions identiques de prise de vue, le seuillage automatique, une fois correctement sélectionné, donne de bons résultats.

Dans le cas de la microscopie par réflexion, cette opération de binarisation requiert en général une opération préalable de rehaussement des contours afin de mieux les mettre en évidence. 
Le seuillage visera alors à sélectionner comme objet ces contours plutôt que la silhouette globale de l'objet.

On effectue ensuite une succession d'opérations sur ces images binaires, et on définit ci-après les opérations morphologiques les plus souvent utilisées dans le domaine des solides divisés. Ces opérations vont avoir pour but de nettoyer l'image de ses derniers petits défauts à savoir :

- objets en contact avec le bord, donc non totalement visibles

- débris

- objets transparents (observés par microscopie à transmission et dont on veut déterminer la silhouette).

\section{3. $\quad$ Mesure :}

Dans le cas des applications traitant de solides divisés, le traitement permet d'isoler dans une image le ou les objets que l'on va ensuite caractériser à l'aide de différents paramètres. Les mesures possibles sont nombreuses et dépendent du problème posé et du but recherché. Les différentes méthodes de quantification morphologique seront aussi abordées [4].

\subsection{Les bases de traitement d'image}

On ne cherchera pas ici à entrer dans les détails des méthodes d'analyse d'images : seules les opérations les plus courantes, utilisées dans le carde de ce travail sont illustrées.

\section{- Seuillage}

Le seuillage est la transformation d'une image en différents niveaux de gris en une image binaire. C'est une opération qui permet de passer de 256 à 2 niveaux de gris, seulement. Le fond d'une image binaire est l'ensemble des pixels de l'image de valeur 0 , et son complément est l'ensemble des pixels de valeur 1 [5].

\section{- Erosion et dilatation}

L'intervention d'un élément structurant B de taille $\lambda$ est essentielle pour l'application de ces deux opérations morphologiques. Il s'agit d'un cercle dans un espace analogique en deux dimensions, et d'un carré de 3 pixels de coté si la trame de numérisation de l'image est carrée. Considérons une image binaire : l'ensemble des pixels objets forme l'ensemble $\mathrm{X}$ de forme quelconque, discontinu ou non. Faisons déplacer l'élément structurant sur l'ensemble de l'image. Pour chaque position posons la question: Est-ce que l'élément structurant est complètement inclus dans l'ensemble X?

La réponse ne peut être que positive ou négative, d'où le nom de transformation en tout ou rien. L'ensemble Y érodé de X par l'élément structurant sera constitué de l'ensemble des 
pixels de X pour lesquels on aura répondu par l'affirmative à la question précédente. La dilatation est l'opération duale de l'érosion; ainsi une dilatation de l'objet $\mathrm{X}$ correspond à une érosion de son complément [5].

\section{- Reconstruction}

La reconstruction est une opération qui met en jeu une érosion puis une dilatation conditionnelle par rapport à l'image initiale : pour chaque pixel devant être mis à 1 par la dilatation de l'érodé, on vérifie son état sur l'image initiale. Si, sur cette dernière, c'est un pixel objet, le pixel de la nouvelle image aura bien la valeur 1 , sinon il restera à 0 [5].

\subsection{Paramètres de quantification morphologique}

\subsubsection{Paramètres de taille}

Diamètre équivalent :

Les paramètres de taille sont obtenus à partir de la silhouette de l'image binaire. La surface, $\mathrm{S}$, est calculée en comptant le nombre de pixels objet dont la valeur est égale à 1 [6].

Le diamètre équivalent est le diamètre du cercle de même surface que la particule (Fig. 1).

$D e q=2 \sqrt{S / \pi}$

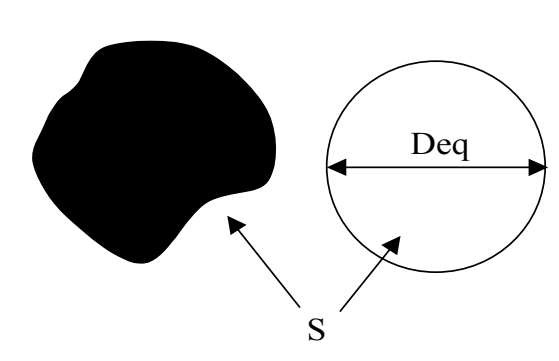

Fig.1. Diamètre équivalent d'une particule

\section{Diamètre de férèt :}

Il correspond à la distance entre les deux tangentes parallèles à une direction donnée et encadrant le contour de la silhouette de l'objet (Fig. 2.a). Les diamètres de férèt horizontal et vertical dépendent de l'orientation de la particule dans l'image. On se sert souvent du diamètre de férèt minimal $\left(F_{\min }\right)$ ' 'largeur de l'objet' et du diamètre de férèt maximal $\left(F_{\max }\right)$ ' 'longueur de l'objet'. Dans le cas d'un rectangle (ou d'un carré) $F_{\max }$ correspond à la diagonale et $F_{\text {min }}$ au plus petit coté [6]. (Fig. 2.b). 
(a)

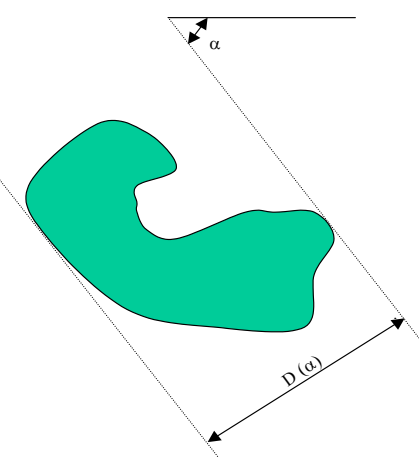

(b)

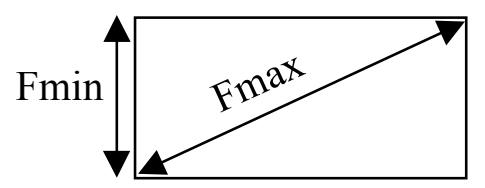

Fig.2. (a) Diamètre de Férèt dans la direction $\alpha$. (b) Diamètre de Férèt dans le cas d'un rectangle.

\subsubsection{Paramètres de forme}

\section{Allongement $\left(\mathrm{F}_{\mathrm{Mm}}\right)$ :}

Le rapport entre le diamètre de férèt maximum et le diamètre de férèt minimum représente un facteur de forme qui exprime l'allongement de l'objet. Ce rapport vaut 1 pour un cercle et 1.41 pour un carré. Plus il est grand, plus l'objet est allongé [6].

$\mathrm{F}_{\mathrm{Mm}}=\mathrm{F}_{\max } / \mathrm{F}_{\min }$

Circularité (C) : La circularité est calculée à partir de la formule suivante : $C=\frac{P^{2}}{4 \pi S}$

où $\mathrm{P}$ est le périmètre et $\mathrm{S}$ est la surface de la silhouette de la particule. Le périmètre est calculé en comptant le nombre de pixels objet " de valeur 1" tel que le voisin est un pixel "de valeur 0 ',

La circularité varie à la fois avec l'allongement de la particule et avec sa rugosité, ce qui peut rendre l'interprétation délicate, si l'on ne prend pas en compte, simultanément, un autre descripteur tel que $\mathrm{F}_{\mathrm{Mm}}$. La circularité est égale à 1 si l'objet est circulaire et supérieur à 1 si l'objet est allongé ou rugueux (Fig. 3). Elle vaut 1.27 pour un carré [6]. 
(a)

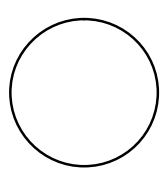

(b)

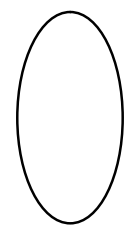

(c)

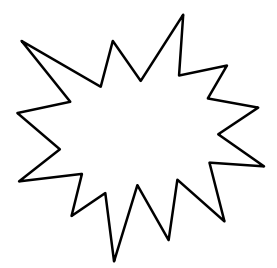

$\mathrm{C}=1$

$\mathrm{C}>1$

$\mathrm{C}>1$

Fig.3. Paramètre de circularité (a) objet circulaire, (b) objet allongé, (c) objet rugueux.

Indice de concavité (IC)

L'enveloppe convexe (E) (Fig.4.b) est obtenue en traçant tous les segments entre les points pivots du contour vectorisé puis en dilatant, remplissant et érodant l'objet ainsi obtenu. La soustraction des images (E) et (M) donne le complément de l'enveloppe convexe par rapport à l'objet [6].

S : aire de la silhouette de l'objet

$\mathrm{S}_{\mathrm{H}}$ : aire de l'enveloppe convexe

$\mathrm{S}_{\mathrm{H}}-\mathrm{S}$ : aire du complément de l'enveloppe convexe

Le facteur de concavité est défini comme étant le rapport entre l'aire de l'objet et l'aire de l'enveloppe convexe. $\mathrm{F}_{\text {conc }}=\mathrm{S} / \mathrm{S}_{\mathrm{H}}$

L'indice de concavité est défini comme étant le rapport entre l'aire du complément par rapport à l'aire du masque de l'objet.

$\mathrm{IC}=\left(\mathrm{S}_{\mathrm{H}}-\mathrm{S}\right) / \mathrm{S}$ 
(a)

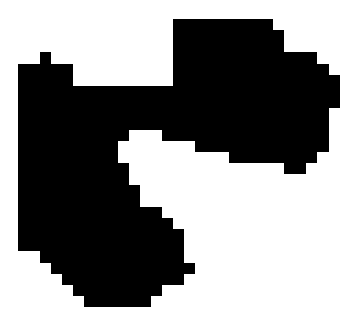

Masque de l'objet

(M) (b)

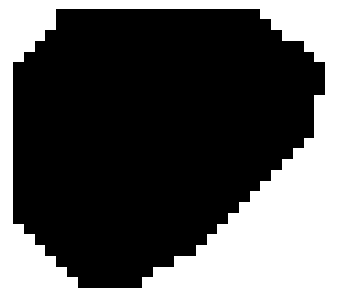

Enveloppe convexe

(E) (c)

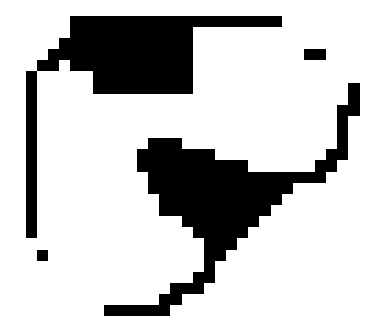

Complément de l'enveloppe convexe : (E) - (M)

Fig.4. L'enveloppe convexe et son complément par rapport à l'objet.

$\underline{\text { Robustesse }\left(\Omega_{1}\right) \text { et plus grande concavité }\left(\Omega_{2}\right)}$

Soit $\omega_{1}$ et $\omega_{2}$ le nombre d'érosions nécessaires pour faire disparaitre complètement l'objet et son complément par rapport à l'enveloppe convexe (Fig. 5) [6].

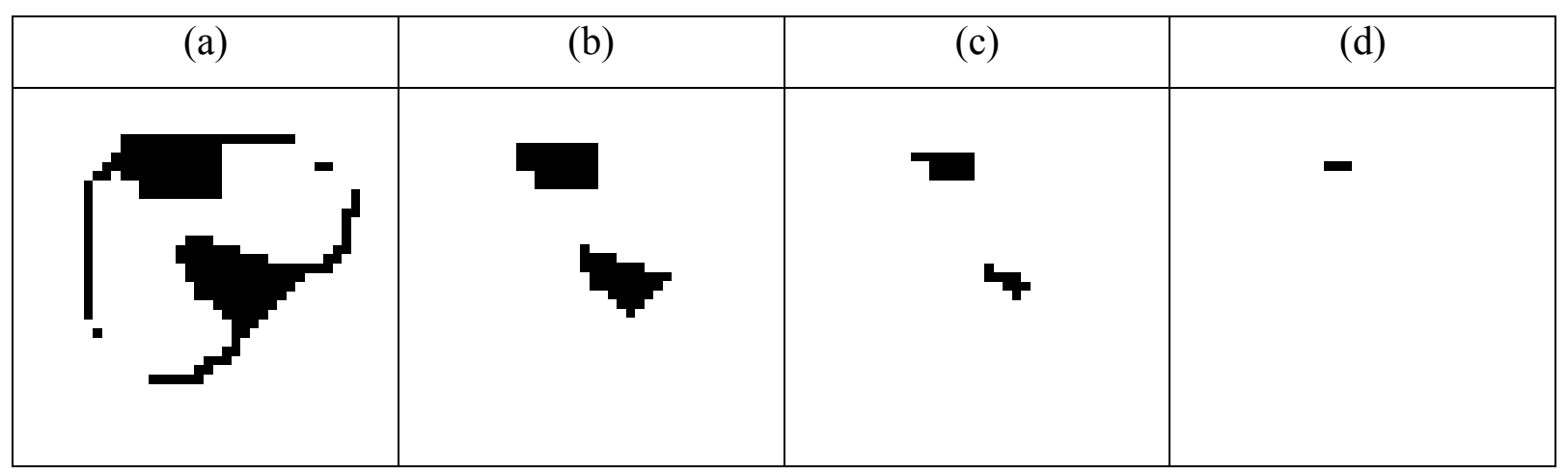

Fig.5. (a) Complément convexe de la Figure 4, (b) après 1 érosion, (c) après 2 érosions, (d) après 3 érosions.

La robustesse ou la compacité de l'objet est définie comme suit :

$\Omega_{1}=2 \omega_{1} \sqrt{S}$

S est la surface de la silhouette de l'objet exprimée en pixels. Pour un carré, $\Omega_{1}$ prend la valeur 1 et pour un objet sous forme de bâtonnet, la valeur de $\Omega_{1}$ est faible. Ce paramètre peut ainsi exprimer l'allongement de particules : il est valable que la particule soit convexe ou concave alors que $\mathrm{F}_{\mathrm{Mm}}$ caractérise l'allongement de l'enveloppe convexe de la particule.

La plus grande concavité est définie comme suit : $\Omega_{2}=2 \omega_{2} \sqrt{S}$ 
Ce paramètre, $\Omega_{2}$, quantifie l'importance de la plus grande concavité de l'objet. Plus $\Omega_{2}$ est proche de 0 , plus la particule est convexe. Par contre, plus $\Omega_{2}$ est important, plus les grosses concavités sont importantes.

\section{CONCLUSION}

Il est nécessaire de disposer le plus précisément possible, des propriétés d'analyses des matériaux (la taille et la forme), Le système d'analyse d'image permet de déterminer la forme des particules, ce dernier paramètre a un effet sur la mesure de la taille de la particule, et donc les résultats obtenus par différents principes de mesure sont fonction de la forme de la particule. Le tableau ci-dessous (Tableau 1) résume l'ensemble des paramètres étudiés.

La forme d'une particule a souvent une influence significative sur les performances du produit final comme la fluidité, l'efficacité d'abrasion, la biodisponibilité. C'est pourquoi il est nécessaire de caractériser la forme d'une certaine manière.

Tableau 1. Récapitulatif des paramètres étudiés

\begin{tabular}{|l|l|}
\hline Paramètre & Equation \\
\hline Diamètre équivalent & $D e q=2 \sqrt{S / \pi}$ \\
\hline Facteur d'allongement & $\mathrm{F}_{\mathrm{Mm}}=\mathrm{F}_{\max } / \mathrm{F}_{\min }$ \\
\hline Circularité & $C=\frac{P^{2}}{4 \pi S}$ \\
\hline Facteur de concavité & $\mathrm{F}_{\text {conc }}=\mathrm{S} / \mathrm{S}_{\mathrm{H}}$ \\
\hline Indice de concavité & $\mathrm{IC}=\left(\mathrm{S}_{\mathrm{H}}-\mathrm{S}\right) / \mathrm{S}$ \\
\hline Robustesse & $\Omega_{1}=2 \omega_{1} \sqrt{S}$ \\
\hline Plus grande concavité & $\Omega_{2}=2 \omega_{2} \sqrt{S}$ \\
\hline
\end{tabular}

\section{REFERENCES}

[1] Brittain H.G., 2001, Particle-size distribution, Part I. Representations of particle shape and size, Pharmaceutical Technology, 25 (12) 38-45.

[2] Pons M.N., Vivier H., Belaroui K., Bernard-Michel B., Cordier F., Oulhana D., Dodds J.A., 1999, Particle morphology : from visualisation to measurement, Powder Technology, $103,44-57$,.

[3] Symons S.J., Fulcher R.G., 1988, Determination of wheat kernel morphological variation by digital image analysis: I. Variation in Eastern Canadian milling quality wheats, Journal of Cereal Science, 8, 211-218.

[4] Pons M.N., Vivier H., Remy J.F., Dodds J.A., 1993, Morphological characterization of yeast by image analysis, Biotechnology and Bioengineering, 42, 1352-1359. 
[5] Pons M.N., Vivier H., Dodds J.A., 1997, Particle Shape Characterization using Morphological Descriptors, Part. Part. Syst. Charact., 14, 272-277.

[6] Belaroui K. (1999), Comprehension des Mecanismes de Fragmentation par Analyse Granulometrique et Morphologique, these de doctorat, INPL, Nancy.

\section{How to cite this article}

Belaroui K and Pons M N. Particle analysis method; morphology by image analysis. J Fundam Appl Sci. 2012, 4(2), 192-201. 